\title{
Sonja Fink "Public Journalism" - ein neues journalistisches Konzept und seine Umsetzung in Lokalredaktionen der USA ${ }^{1}$
}

"In the Seventies the society needed us to be watchdogs, critical of the system.

I was very inspired by that. But today there is something else being asked of us, in addition to the watchdog role: we have to do our job in a way that allows people to do their job as citizens."

John Dinges, editorial director of National Public Radio

\section{Ausgangspunkt und Fragestellung}

Die Landschaft der Printmedien in Deutschland: Schon lange blüht sie nicht mehr so, wie es Journalisten und Verleger gerne sähen. Schneller, bunter, boulevardesker soll sie sein, die Nachrichtenvermittlung im Zeitalter des Häppchen-Journalismus. Zuwachsraten lassen sich da höchstens noch bei zusätzlichen Online-Angeboten auf der Datenautobahn im Internet verzeichnen. Insbesondere im Bereich der Tageszeitungen hält die Dürreperiode in Form von stagnierenden Stückzahlen - was in entsprechendem Kontext dann sogar als Erfolg vermarktet wird - und stetigem Auflagenverlust jedoch nach wie vor an, wie auch die Zahlen der aktuellen IVW-Auflagenliste belegen. ${ }^{2}$ Auf Zeitungskongressen sind sich die Fachleute sodann trotz aller Ratlosigkeit, wie das Produkt Tageszeitung rundum erneuert und kunden-, sprich leserfreundlicher gestaltet werden könne, in folgendem Punkt einig: Die Zukunft der Zeitung liegt im Lokalen. Lutz Glandt, Geschäftsführer der „Neuen Westfälischen“, formulierte es auf diese Weise:

„Es geht also nicht darum - das ist die Quintessenz -, neue Produkte für neue Zielgruppen zu erfinden, sondern das Bestehende zu verbessern: eine Aufgabe auch der Redaktionen. Die müssten wieder öfter rausgehen

1 Dem hier abgedruckten Aufsatz von Sonja Fink liegt eine Diplomarbeit im Fachgebiet Journalistik an der Katholischen Universität Eichstätt zugrunde, die im Oktober 1999 bei Prof. Dr. Walter Hömberg entstand.

2 Vgl.: IVW-Auflagendaten Quartal II/1999, in: http://www.zeitungen-online.de/ auflagen. 
und die Stimmung der Menschen vor Ort ausloten, den Terminjournalismus aufgeben und selbst aktiv werden, Stellung beziehen und Konflikte moderieren, viele eigene Geschichten und weniger Agenturmaterial bringen, transparenter arbeiten und mit den Lesern besser kommunizieren $[. ..] . " 3$

Und Hans-Jürgen Hippler, Forschungsleiter der Zeitungs-MarketingGesellschaft, setzte noch eins drauf:

„Die Zeitung muss als Institution vor Ort sichtbar sein. Themen müssen selbst recherchiert, Diskussionen über kontroverse Themen angeregt werden. Es gilt, sich unabhängig zu machen von lokalen Honoratioren in Politik und Vereinen. Deren Interessen decken sich nicht notwendigerweise mit denen der Leser." 4

Welche Bedeutung hat nun diese Einschätzung für die Zukunft des Printjournalismus?

Der Blick nach Amerika, wo man den hiesigen Trends meist um rund zehn Jahre vorauseilt, zeigt, wohin die Entwicklung dort bereits geführt hat: "Public Journalism“ lautet das Credo, mit dem zunächst einzelne Journalisten in Zusammenarbeit mit ihren Verlegern Ende der 80-er/ Anfang der 90-er Jahre im Rahmen von zeitlich begrenzten Projekten wie beispielsweise bei Wahlkampagnen versucht haben, neue Ansätze in der Art und Weise der Berichterstattung zu finden. Dabei geht es im Wesentlichen darum, die verloren gegangene Beziehung zwischen dem Journalismus, dem Leser und seiner Region, seiner community, wieder herzustellen. Denn insbesondere in dieser Verknüpfung sehen die Vorreiter des „Public Journalism“ die grundlegende Bedeutung für eine funktionierende Demokratie. Wie kann man Menschen dazu veranlassen, wieder am öffentlichen Meinungsbildungsprozess teilzunehmen?, 5 heißt die zentrale Fragestellung hinter der konkreten Umsetzung ihrer Idee. Damit heben die Vordenker dieser Bewegung die Bedeutung des Journalismus auch im lokalen Bereich auf eine gesamt-gesellschaftliche Ebene und binden den Berichterstatter und seine Arbeit in die Verantwortung für den Erfolg des Zusammenlebens in der Gemeinschaft mit ein.

"Doing Public Journalism“, das heißt infolgedessen, sich zunächst

3 Lutz Glandt im Rahmen seines Referats über die Zukunft der Zeitung auf dem Zeitungskongress der Initiative Tageszeitung und der Bundeszentrale für politische Bildung unter dem Tagungsmotto "Zeitung ist Zukunft" im Mai 1998 in Bielefeld.

4 Hippler, Hans-Jürgen: Was wollen die Leser?, in: Insight. NeueMedienMärkteMacher, Heft 8/98, S. 10.

5 Vgl. Fürsich, Elfriede: Civic Journalism. Debatte über berufliches Selbstverstăndnis, in: Informationsdienst der Deutschen Gesellschaft für Publizistik- und Kommunikationswissenschaft (Hg.): Aviso, No.15/November 1995, S. 12. 
einmal von der Tradition des ausschließlich „objektiven“, unbeteiligten Berichterstattens zu verabschieden; nicht nur Missstände aufzudecken, sondern ebenso selbstverständlich auch zu versuchen, zusammen mit den zuständigen Gremien, Behörden oder Einzelpersonen eine Diskussion über mögliche Lösungsansätze in Gang zu bringen. Gemeindeversammlungen und Telefonaktionen auf Initiative der lokalen Zeitung stehen dann ebenso auf dem Programm wie ein monatliches Leserforum oder ein Leitartikel, der den Leser ganz direkt zum Handeln in seiner Gemeinde auffordert. Außerdem gilt es, den nur allzu bequemen ElitenJournalismus, bei dem man vom Schreibtisch aus zu bestimmten Sachfragen nur die immer wieder gleichen Experten und offiziellen Informationsträger befragt, aufzugeben. Denn genau dadurch sei, so der Vorwurf der "Public Journalism"-Pioniere, eine Sphäre der Öffentlichkeit entstanden, die nur noch auf einer Ebene zwischen Journalisten und Experten vorbei am Leser - stattfinde.

Vor diesem Hintergrund erschien es überaus spannend, sich diesem noch recht jungen, bislang weder im deutschen Journalismus noch in der Kommunikationswissenschaft entsprechend untersuchten Phänomen „Public Journalism" systematisch zu nähern und anhand von untersuchten Einzelfällen eine empirisch fundierte Bestandsaufnahme der diesbezüglichen Situation in Amerika zu liefern. Die Frage nach der Relevanz des Themas auch für den deutschen Journalismus ergibt sich quasi automatisch: Sind, oder waren vielleicht, - beispielsweise während der 70 -er Jahre in Form des anwaltschaftlichen Journalismus -, ähnliche Denkansätze nicht auch hierzulande immer wieder zu finden? Denn dass man, wie eingangs beschrieben, derzeit auf vielfältige Weise nach neuen Formen in der Berichterstattung sucht, ist evident. Relaunches, Redesigns, Redaktionsmarketing und projektbezogenes Outsourcing seien hier nur als Schlaglichter einiger zumindest in ihrer Außenwirkung oft wenig zielgerichtet erscheinenden Zeitungsreform-Experimente in Deutschland genannt.

\subsection{Theoretischer Ansatz und Forschungskontext}

Auf den ersten Blick relativ lose erscheint die theoretische Verankerung der "Bewegung“. ${ }^{6}$ Jay Rosen, Journalistik-Professor an der New York University und Doktor der Philosophie, hat sich als einer der wenigen Akademiker, die sich überhaupt mit „Public Journalism“ auseinanderge-

6 Vgl. auch: Haas, Tanni: What's „Public" About Public Journalism? Public Journalism and the Lack of a Coherent Public Philosophy. In: Communication Theory, 9. Jahrgang 1999/Nr. 3. August 1999, S. 346 ff. 
setzt haben, am deutlichsten profiliert. Mittlerweile gilt er aufgrund der von ihm herausgearbeiteten politiktheoretischen wie auch systemtheoretischen Aspekte und nicht zuletzt der berufsethischen Perspektive, durch die er einer zunächst recht diffusen Entwicklung eine zumindest in Ansätzen einheitliche Form und nicht zuletzt auch ihren Namen gab, als „Vater" des „Public Journalism". Seither spielt sich die lebhafte Forschungsdiskussion hauptsächlich in Form von Aufsätzen diverser Berufspraktiker in den einschlägigen amerikanischen Fachzeitschriften ab. Dabei rücken berufsethische Überlegungen und die Frage nach der Selbstreferenz oft in den Mittelpunkt der thematischen Auseinandersetzung. Und nicht zuletzt spielt auch die aktuelle Kommunitarismus-Debatte und die darin mitunter thematisierte Rollenverteilung von politischen Eliten und der Presse eine entscheidende Rolle im Konzept vom Zusammenspiel zwischen Journalismus und Öffentlichkeit. Bei der Einordnung des Phänomens „Public Journalism" in den deutschen Forschungskontext erscheint in Anbetracht der Problematik etwa von beruflichem Rollenverständnis, Objektivitätsanspruch und Glaubwürdigkeit vor allem der Bezug zur Journalismusforschung interessant. Um eine systematische Vorgehensweise zu gewährleisten und gleichzeitig den Rahmen der Untersuchung abzustecken, hat die Autorin zunächst folgende forschungsleitende Fragen formuliert:

$F_{1}$ : Wo liegen die geistigen Wurzeln des „Public Journalism"?

$F_{2}$ : Worin besteht die Notwendigkeit für ein neues journalistisches Konzept?

$F_{3}$ : Auf welche Weise unterscheidet sich „Public Journalism" vom bisher praktizierten Journalismus?

$F_{4}$ : Beschränkt sich die Umsetzung von „Public Journalism“ in der Praxis auf Projekte in lokalen und regionalen Zeitungen?

$F_{5}$ : Geht mit dieser Entwicklung eine Veränderung im Berufsverständnis der Journalisten einher?

F $_{6}$ : Hat sich der „Public Journalism“ seit seinen Anfängen vor zehn Jahren bis heute verändert?

$F_{7}$ : Hat „Public Journalism" Auswirkungen auf die redaktionellen Strukturen von Zeitungen, die sich der Bewegung anschließen?

$F_{8}$ : Wie wird „Public Journalism" von den Lesern aufgenommen?

$F_{9}$ : Werden durch „Public Journalism“ neue Zielgruppen erreicht?

$F_{10}$ : Ist der Erfolg/Misserfolg von „Public Journalism" überhaupt messbar?

$F_{11}$ : Ist „Public Jorunalism“ möglicherweise nichts weiter als eine PR-Strategie?

$F_{12}$ : Ist „Public Journalism" ein rein amerikanisches Phänomen? 


\subsection{Methodik der Studie}

Es bedarf mehrerer empirischer Forschungsinstrumente, um sich dem Thema „Public Journalism" in seiner Vielschichtigkeit zu nähern. Ausgehend von einer überwiegend theoretischen Betrachtung über die gegenseitige Bedingtheit von Öffentlichkeit und Demokratie, die sich, angereichert mit einigen Ergebnissen ausführlicher mündlicher Befragungen, auf eine eingehende Literaturanalyse stützt und gewissermaßen einen historisch-gesellschaftspolitischen Kontext für die aktuelle Entwicklung liefert, wurden exemplarisch einige „Public Journalism“-Projekte aus unterschiedlichen Bereichen der aktuellen Redaktionsarbeit auf die spezielle Fragestellung hin untersucht. Diese Projektbeschreibungen, denen sowohl Artikel aus den relevanten amerikanischen Fachzeitschriften als auch Recherchen vor Ort zugrunde liegen, wurden ebenso wie die Literaturanalyse ergänzt durch leitfadengestützte Intensiv-Interviews - die hauptsächliche Methodik der Untersuchung. Die Befragung als formalisiertes Instrument der empirischen Sozialforschung erschien dabei in Form persönlicher Interviews mit Hilfe eines zuvor formulierten Katalogs offener Fragen am geeignetsten, da diese sich insbesondere bei Problemstellungen anbietet, „fïr die nicht schon umfassende Kenntnisse über das Universum möglicher Antworten vorhanden sind". ${ }^{7}$ Diese Gespräche wurden „zur Klärung von Zusammenhängen "8 sowohl mit den Pionieren des „Public Journalism“, als auch mit Redakteuren, die an Projekten beteiligt waren oder es auch heute noch sind, sowie mit Menschen, über die in Form von „Public Journalism" berichtet wurde, und nicht zuletzt auch mit Kritikern der Bewegung, geführt. Deduktiv abgeleitet aus Literaturanalyse und Interview-Auswertung erfolgte die Hypothesen-Formulierung. Die Hypothesen wurden anhand einer quantitativen Inhaltsanalyse auf ihre Gültigkeit hin überprüft. Diese Forschungstechnik, „mit der man aus jeder Art von Bedeutungsträgern durch systematische und objektive Identifizierung ihrer Elemente Schlüsse ziehen kann, die über das einzelne analysierte Dokument hinaus verallgemeinerbar sein sollen", setzte sich im vorliegenden Fall zum einen in komparativer Art und Weise mit der Wahlkampf-Berichterstattung einer ausgewählten Lokalzeitung in den Jahren 1988, 1992 und 1996 auseinander. Zum anderen beschäftigte sie sich zur thematischen Abrundung aber auch mit zwei unterschiedlichen Formen der Sozialberichterstattung, die unter dem

7 Kromrey, Helmut: Empirische Sozialforschung, Opladen 51991, S. 279.

8 Atteslander, Peter: Methoden der empirischen Sozialforschung, Berlin/New York 81995 , S. 171.

9 Kromrey, Helmut: a.a.0., S. 232. 
Vorzeichen des „Public Journalism" entstanden sind. Durch die Entscheidung für eine inferentielle Analyse, ${ }^{10}$ eine spezielle, weitergefasste Form der traditionellen quantitativen Inhaltsanalyse, sollte bereits die Auswahl dieser Methodik signalisieren, dass außer den rein quantitativen statistischen Kennwerten bei der Analyse des Datenmaterials auch Informationen und Aussagen qualitativer Art vermerkt wurden und dementsprechend als ergänzendes Element in die Auswertung einflossen.

\section{Das Verhältnis von Demokratie und Öffentlichkeit}

„Public Journalism is nothing more than the conviction that journalism's business is about making citizenship work. [...] Actually, public journalism has a golden rule, and just as easy to elaborate into a code book of professional norms: Journalism should advocate democracy without advocating particular solutions."

Arthur Charity, Fachautor und Mitarbeiter des "Project on Public Life and the Press"

Die Ursprünge der „Public Journalism“-Bewegung erscheinen ebenso vielfältig wie uneinheitlich. Die Bemühung, eine stringente Ableitung sei es aus der Kommunikationsgeschichte, sei es aus der Politiktheorie abzuleiten, erweist sich als mühevolles und nur an den wenigen, eben aufgezeigten Anknüpfungspunkten von Erfolg gekröntes Unterfangen. „Public Journalism“ ist eben „an idea that just happened“.11 Dennoch lässt sich zur Beantwortung von $F_{1}$, der forschungsleitenden Frage nach den geistigen Wurzeln des „Public Journalism“, ein zumindest grobmaschiges Beziehungsmuster zwischen den theoretischen Abhandlungen einiger, überwiegend aus der Gegenwartsforschung stammender Soziologen, Philosophen und Politikwissenschaftler und dem zentralen Anliegen

10 Inferentielle Inhaltsanalyse: Stellt insoweit eine Erweiterung des deskriptiven Ansatzes dar, "als sie davon ausgeht, dass bestimmte inhalts-inteme Merkmalsausprägungen mit bestimmten inhalts-externen Merkmalsausprägungen korrelieren. Inferenz heißt dabei, dass bestimmte Merkmale eines Textes mit bestimmten Merkmalen eines Kontextes zusammenhängen [.../“. Vgl. Merten, Klaus: Inhaltsanalyse. Einführung in Theorie, Methode und Praxis. Opladen 1983, S. 23.

11 Rosen, Jay: The Action of the Idea: Public Joumalism in Built Form, in: Glasser, Theodore ( $\mathrm{Hg}$.): The Idea of Public Journalism. Auszug aus einem noch unverbffentlichten Buchmanuskript, das der Autorin freundlicherweise zur Verfügung gestellt wurde, New York 1999, S. 1. 
des „Public Journalism" herstellen: Ziel sowohl der auf die antike Form der Polis und der jüngst von den Kommunitaristen wieder aufgenommenen Idee der Basisdemokratie, als auch dieser neuen Form des anwaltschaftlichen Journalismus ist das aktive Mitgestalten der demokratischen Gesellschaft durch ein re-politisiertes Klima, in dem die Medien künftig nicht mehr nur als mitunter sogar zynische Beobachter fungieren, sondern sich selbst als verantwortliche Mitgestalter der Gemeinschaft begreifen. Weg vom Eliten-Journalismus, der fern von den Interessen und Bedürfnissen der Leser, nur noch dem Selbstzweck frönend, in Kungeleien mit Politik und Wirtschaft ein immer bedeutungsloseres Dasein fristet, lautet das Credo. Denn welche Rolle den Journalisten zugebilligt würde, wenn sie nicht selbst Initiative ergriffen, zeigt folgende Aussage in beispielhafter Weise: „Those who exclude journalism from the broader scholarly and political discussion about the restoration of community and citizenship are sending the profession a message: You may be part of the problem, but you are not considered part of the solution." 12

\section{Zehn Jahre "Public Journalism" in den USA}

It is time for those of us in the world's freest press to become activists, not on behalf of a particular party or politicians, but on behalf of the process of self-government.

David Broder, Veteran „Washington Post“ political reporter

Was sich aus dem Aufbruch einiger "Pioniere" sowohl aus der kommunikationswissenschaftlichen Theorie als auch aus der redaktionellen Praxis im Laufe der vergangenen zehn Jahre entwickelt hat, welche Projekte im Zeichen des „Public Journalism" entstanden sind, welche Auswirkungen das beispielsweise auf die redaktionelle Organisation und das journalistische Selbstverständnis hatte, welche Probleme auftraten und welche Kritik aus was für Gründen geäußert wurde - all diesen Fragen und anderen Aspekten mehr wurde anhand von exemplarisch ausgewählten Projekten in Wichita/Kansas, New York, Washington D.C. und Charlotte/North Carolina im Rahmen von 13 Intensiv-Interviews nachgegangen. Deren Auswertung führte zusammen mit den Ergebnissen der umfangrei-

12 Anderson, Rob/Dardenne, Robert/Killenberg, George M.: The American newspaper as the public conversational commons, in: Black, Jay: Mixed News. The Public/ Civic/Communitarian Journalism Debate, Mahwah, 1997, S. 102. 
chen Literaturanalyse zu folgenden Antworten auf die eingangs gestellten Forschungsfragen:

- Worin also besteht die Notwendigkeit für ein neues journalistisches Konzept?

Dafür waren wohl maßgeblich zweierlei Gründe ausschlaggebend: Zum einen spürten die Journalisten im Laufe der Zeit eine starke Unzufriedenheit mit der Aufgabe, die sie in der Gesellschaft zu spielen hatten. Die Glaubwürdigkeit der Medien sank stetig, und die vermittelten Informationen und Meinungen besaßen ganz offensichtlich nur noch geringfügige Relevanz. Stattdessen hatten sich die Journalisten ins gesellschaftliche Abseits manövriert als - wie sie glaubten - „objektiven Berichterstatter", die jedoch dadurch vielmehr zum Verlautbarungs-Instrument der Eliten geworden waren und dabei die Verbindung zu den Durchschnittsbürgern, das Gespür für die Themen der Menschen, verloren hatten.

Als weiterer auslösender Faktor darf sicherlich auch die ökonomische Situation der Zeitungen nicht außer Acht gelassen werden. Auflagenverluste und multi-mediale Konkurrenzangebote zwangen die Verlagshäuser zum Umdenken und zur Entwicklung neuer Konzepte. „Public Journalism" ist eines von ihnen.

- Inwiefern unterscheidet sich - in Anlehnung an $F_{3}$ gefragt - nun "Public Journalism" von der bisherigen Berichterstattung?

Bei „Public Journalism" beschränkt sich das journalistische Berufsverständnis nicht ausschließlich auf die Rolle des objektiven, unbeteiligten Beobachters, sondern der Journalist sieht sich selbst als aktives Mitglied der Gesellschaft. Es geht bei einer davon geprägten Berichterstattung nicht mehr nur darum, Missstände aufzudecken und die Leser darauf hinzuweisen, sondern zusätzlich darum, gemeinsam mit der Bevölkerung Lösungskonzepte zu entwickeln. Konkrete Projekte haben bislang überwiegend während eines Wahlkampfes oder zu sozialen Brennpunkt-Themen stattgefunden. Dabei unterschied sich die "Public Journalism"-Berichterattung auf folgende Weise: Während der Wahlkampagne wurde die Agenda nicht mehr ausschließlich von den Kandidaten und ihren Managern bestimmt, sondern die Zeitung führte eigene Erhebungen durch und veranstaltete Diskussionsrunden, um herauszufinden, welche Sachthemen den Bürgern gerade besonders wichtig erschienen. $\mathrm{Zu}$ diesen Themen wurden dann Hintergrundberichte und Analysen verfasst sowie Stellungnahmen der Kandidaten eingeholt. Zudem versuchte sie, den Lesern die Bedeutung ihrer gesellschaftspolitischen Aufgabe, wählen zu gehen, deutlich zu machen.

Auf sozialem Gebiet engagierten sich die Verfechter der „Public 
Jorunalism"-Idee überwiegend in Sachen Kriminalitätsbekämpfung, Rassenkonflikte und Stadtplanung, aber auch im Bereich der Wirtschaftsförderung. Durch ein solches gesellschaftliches Engagement versuchen die Zeitungen, die verlorengegangene Glaubwürdigkeit bei den Lesern zurückzugewinnen und somit natürlich auch positive Impulse in Richtung Auflagenentwicklung auszusenden.

Die meiste Kritik an dieser "anderen“ Berichterstattung entzündet sich daran, dass der Journalist sich nun, wie immer wieder angemerkt wird, nicht mehr dem Objektivitäts-Maßstab verpflichtet fuihle, sondern jetzt auch deutlich Partei für etwas ergreife, zum Agenda-Setter werde und dadurch bestimme, was die Leute zu wissen bekommen und was nicht.

- Beschränkt sich die Umsetzung von „Public Journalism“ in der Praxis auf Projekte in lokalen und regionalen Zeitungen? $\left(\mathrm{F}_{4}\right)$

Berücksichtigt man bei der Untersuchung dieser Frage nur diejenigen Projekte, Serien und Einzelartikel, die tatsächlich mit dem Label "Public" oder "Civic Journalism" versehen worden sind, oder zumindest auf irgendeine Art als solche kenntlich gemacht wurden, dann muss man diese Frage für den heutigen Zeitpunkt wohl bejahen. Bezieht man jedoch die Aussagen amerikanischer Journalisten über die Entwicklung auf dem bundesweiten Zeitungsmarkt in die Betrachtung ein, und blättert man hin und wieder auch in den "Großen" wie der "New York Times" oder der "Washington Post", dann lässt dies eigentlich nur eine Schlußfolgerung zu: Die Probleme, die Zeitungsmacher derzeit plagen - vom Auflagen- und Glaubwürdigkeitsverlust bis zur Überalterung der Leserschaft - sind überall die gleichen, ob in der Großstadt oder in der Provinz. Aufgrund des überschaubareren Rahmens und der größeren Experimentierfreudigkeit in kleineren Gemeinden entwickelte sich "Public Journalism“ nicht zuletzt ja auch in Anlehnung an den kommunitaristischen Gedanken zwar aus dem kleinen und mittleren Marktsegment heraus, Grundzüge der Idee lassen sich aber - wenn auch nicht explizit unter diesem Namen - inzwischen auch in der Berichterstattung der nationalen Zeitungen erkennen.

- Geht mit dieser Entwicklung eine Veränderung im Berufsverständnis der Journalisten einher? $\left(\mathrm{F}_{5}\right)$

Auf jeden Fall. Die „Public Journalism“-Bewegung entwickelte zwar keine eigenen, verbindlichen Richtlinien, doch immerhin führten einige damit verbundene Vorstellungen zu einer dementsprechenden Änderung des Ethik-Codexes der Society of Professional Journalists. $\mathrm{Zu}$ den allgemein anerkannten Wertvorstellungen im „Public Journalism" zählen Ausgewogenheit, Fairness und Vollständigkeit, Aufrich- 
tigkeit, Authentizität und Glaubwürdigkeit. Außerdem sollten die Redakteure eine Leitbildfunktion innerhalb der Stadt einnehmen, die Nachrichten erklären und gewichten sowie für die Leser erreichbar sein. Der Journalist versteht sich als Überbringer von Nachrichten und Meinungen, als Erklärer, Beobachter, Anwalt, Vermittler, Teilnehmer und kritischer Analyst. Ob damit auch die Aufgabe verbunden sei, den Lesern Lösungen für ihre Probleme direkt vorzulegen, ist umstritten. Zumindest die Theoretiker des „Public Journalism" lehnen diese Art von Einmischung in gesellschaftliche Prozesse ab und empfehlen vielmehr, mit den Lesern im Dialog Lösungsvorschläge $z u$ entwickeln.

- Hat sich der "Public Journalism" seit seinen Anfängen vor zehn Jahren bis heute verändert? $\left(\mathrm{F}_{6}\right)$

Sicherlich. Aus dem zunächst theoretisch-analytischen Nachdenken über das aktuelle Verhältnis und die damit verbundenen Probleme zwischen Journalisten, Lesern und Politikern entwickelten sich die ersten redaktionellen Projekte, die bald über das ganze Land verteilt sowohl gute als auch schlechte Nachahmer fanden. Inzwischen scheint sich der Trend weg vom Einzelprojekt hin zur alltäglich anwendbaren Berufsphilosophie in den Redaktionen durchzusetzen. Das ist finanziell und personell weniger aufwendig, entspricht aber im Prinzip ganz den Vorstellungen, die „Public Journalism"-Begründer Jay Rosen von der Bewegung hatte. Nach der "Learning by doing"-Phase konzentriert sich die Beschäftigung und Weiterentwicklung der Idee nun vor allem auf die Aus- und Weiterbildungseinrichtungen an den Universitäten sowie auch im Ausland.

- Hat „Public Journalism“ Auswirkungen auf die redaktionellen Strukturen von Zeitungen, die sich der Bewegung anschließen? $\left(\mathrm{F}_{7}\right)$

Nein, personelle Veränderungen sind nur in den seltensten Fällen, und das zumeist nur während der Anfangsphase, festzustellen. Die „Public Journalism“-Bewegung profitierte jedoch sicherlich von einer Aufweichung der Ressortgrenzen zugunsten der Ausbildung themenbezogener Teams, die bereits zuvor eingesetzt hatte. Das heißt, dass für die Bearbeitung bestimmter Themen - wie beispielsweise die Lage in einem bestimmten Stadtteil - kleine Teams von drei bis höchstens vier Redakteuren aus meist unterschiedlichen Sachgebieten für einen befristeten Zeitraum zusammen an diesem Projekt arbeiten und vom Alltagsgeschäft weitgehend freigestellt sind.

Wenn für manche Projekte vorübergehend zusätzliches Personal beschäftigt wurde, so handelte es sich dabei nicht um Journalisten, sondern um sogenannte Koordinatoren, die sich um die organisatorische Abwicklung der Aktionen kümmerten und als Bindeglied 
zwischen Lesern und Redaktion betrachtet wurden. Da inzwischen jedoch - wie bereits an anderer Stelle ausführlich beschrieben kaum noch solche groß angelegten Projekte ins Leben gerufen werden, sondern Elemente des „Public Journalism" in die Alltagsberichterstattung Eingang finden, hat das auf die personellen Struktu. ren keinerlei Auswirkungen.

- Wie wird "Public Journalism" von den Lesern aufgenommen? $\left(\mathrm{F}_{8}\right)$ $\mathrm{Da}$ gibt es die einen, das sind überwiegend Kandidaten für politische Ämter, Behördenleiter und offizielle Informationsträger an der Spitze einer Verbands oder einer Vereinigung, die sich von dieser Art der Berichterstattung nicht mehr in ausreichendem Maße vertreten fühlen und ihre Kritik auch öffentlich äußern. Und dann gibt es auf der anderen Seite eine ganze Vielzahl von Bürgern, die in Leserbriefen und auf Veranstaltungen ihre Zustimmung dazu bekundet, dass endlich einmal jemand ein Auge darauf hat, was in der Stadt eigentlich vor sich geht und warum manche Zustände so sind, wie sie sind. Die enorme Resonanz auf Projekte wie beispielsweise "Taking back our Neighborhoods" habe gezeigt, so die „Public Journalism“. Anhänger, wie groß die Hilfsbereitschaft unter den Menschen sei, welche kreativen Ideen aus der Bevölkerung kämen und wie viel man erreichen könne, wenn man den Leuten ein Fonum dafür biete und sie bei der Organisation anleite. Hunderte von Zuschriften, E-Mails, Faxen und Anrufen nicht nur beim "Charlotte Observer", sondern auch im Rahmen anderer Projekte sprechen dafür, dass die „Public Journalism"-Idee und ihre konkrete Umsetzung durch die jeweilige Redaktion bei den Lesern überwiegend positiv aufgenommen wurde.

- Werden durch „Public Journalism“ neue Zielgruppen erreicht? $\left(F_{9}\right)$ Ja und nein. Die Redakteure bekunden zwar vielfach, dadurch eine ganz erhebliche Anzahl neuer Informanten hinzugewonnen zu haben, doch ob deren Informationsverhalten dann auch längerfristig über das punktuelle Rezipieren während eines bestimmten Zeitraumes hinausgeht, darf wohl zurecht bezweifelt werden. Offensichtlich geht es im „Public Journalism“ weniger darum, neue, andere Zielgruppen als Leser zu erschließen, als vielmehr darum, die Glaubwürdigkeit bei den „alten" Lesern dadurch zu erhöhen und die Leser-Blatt-Bindung zu verstärken, indem man neue Zielgruppen als Handlungsträger für die Berichterstattung entdeckt.

- Ist der Erfolg/Misserfolg von „Public Journalism“ überhaupt messbar? $\left(\mathrm{F}_{10}\right)$

Laut Jan Schaffer, der Leiterin des „Pew Center for Civic Journalism", gibt es ein ebenso einfaches wie eindeutiges Indiz für den Erfolg von „Public Journalism"-Aktionen: Die Resonanz der 
Bevölkerung. Zudem gibt es bleibende Ergebnisse der Bemühungen, wie beispielsweise ein Freizeitheim im Charlotter Stadtteil Seaversville, das im Zuge von "Taking back our neighborhoods" errichtet wurde.

Eine andere, etwas aufwendigere Methode, um zu ermitteln, ob die Bemlihungen der Journalisten nicht etwa doch ins Leere gingen, stellen Umfragen dar, die im zeitlichen Abstand von mehreren Monaten unter der Bevölkerung der Stadt gemacht wurden, in der die Zeitung "Public Journalism"-Projekte ins Rollen gebracht hatte. Dabei wurden die Leute, nachdem sie die Einstiegsfrage, ob ihnen der Name des jeweiligen Projekts überhaupt etwas sage, auch nach folgenden Dingen gefragt: ob sie jetzt mehr über Politik nachdenken würden; ob sie ärgerlich seien über die Vielzahl derjenigen Menschen, denen das Wohlergehen der Stadt völlig gleichgültig sei; ob sie eine bessere Idee hätten, wie man die Probleme hier angehen könne; ob sie stärker eingebunden werden möchten in die Veränderungen in der Stadt; und nicht zuletzt auch, ob sie sich nun stärker verpflichtet fuihlten, zur nächsten Wahl zu gehen als bisher.

Die Ergebnisse dieser Umfragen malen zwar ein überaus positives Bild von dem mutmaßlich deutlichen Einfluss der „Public Journalism"-Berichterstattung auf die Einstellung der Menschen zu ihrer Stadt und hinsichtlich ihres gesellschaftspolitischen Engagements: Angeblich denken 59 Prozent der Leute in Charlotte jetzt mehr über Politik nach, 72 Prozent sind wiitend auf andere Leute, denen die Bedürfnisse der Stadt und ihrer Bewohner gleichgültig sind, 78 Prozent wollen in Zukunft aktiv an der Umgestaltung mitarbeiten, 86 Prozent können sich nun eine bessere Vorstellung von bestimmten Problemen machen, und 67 Prozent fühlen sich mittlerweile stärker verpflichtet, bei der nächsten Wahl ihre Stimme abzugeben. ${ }^{13}$ Doch selbst Liz Chandler, leitende Redakteurin eben jenes Projekts, äußerte Zweifel daran, ob die Zeitung über einzelne Erfolgsmeldungen hinaus auch langfristig etwas im Sinne der benachteiligten Bevölkerung in den armen Teilen der Stadt habe erreichen können. Der Erfolg für die Zeitung im wirtschaftlichen Sinne, der sich im Wesentlichen an der Auflagenentwicklung zu messen hat, ist heute jedoch noch nicht zu beurteilen. Zwar deuteten auch kurze Auflagensteigerungen während konkreter Projektphasen und kurz danach auf eine positive Ausstrahlung hin, doch da inzwischen „Public Journa-

13 Vgl. Thorson, Esther: Evaluation Summary, in: Civic Lessons. Report on Four Civic Journalism Projects Funded by The Pew Center for Civic Journalism, Philadelphia 1997, S. 4. 
lism" als kontinuierliches Element der Bericherstattung praktiziert wird, muss die längerfristige Entwicklung beobachtet werden. Im übrigen läßt sich ein über Jahre hinweg vollzogener Glaubwïrdigkeitsverlust gewiss nicht in wenigen Wochen kompensieren. In kontinuierlichen zeitlichen Abständen erhobene Daten über das Image der Zeitung in Kombination mit statistischen Beobachtungen über die aktive Bürgerbeteiligung können nach Einschätzung der Autorin aber sicherlich als zumindest ein aussagekräftiges Indiz für den Erfolg oder Misserfolg von „Public Journalism"-Bemühungen dienen.

- Ist „Public Journalism“ möglicherweise nichts weiter als eine PRStrategie? $\left(\mathrm{F}_{11}\right)$

Darüber gingen die Meinung lange Zeit stark auseinander. Während von den Kritikern angeführt wird, einzig die ökonomische Situation der Zeitungen habe überhaupt erst dazu geführt, neue Konzepte zu entwickeln, sehen das die "Pioniere“ wie Jay Rosen und Davis Merritt erwartungsgemäß anders. Kann aber tatsächlich von einer versteckten PR-Strategie gesprochen werden, wenn mit einem messbaren Erfolg erstens höchstenfalls langfristig und zweitens wohl weniger in Form von Auflagenzuwachsraten als vielmehr erhöhten Glaubwïrdigkeitswerten $z \mathrm{u}$ rechnen ist? Bewertet man in Zeiten harter Konkurrenz - von der aber zum Beispiel weder in Charlotte, noch in Wichita, noch in Madison auf dem Medien-Sektor wirklich die Rede sein kann - aber bereits die Stagnation als wirtschaftliches Plus, wäre diese Argumentation kaum zu halten.

Sicherlich darf man nicht die Augen davor verschließen, dass „Public Journalism" nur allzu bereitwillig genügend Ansatzpunkte liefert, um in der Hoffnung auf schnelle Zuwachsraten als Werbekampagne zweckentfremdet zu werden. Es erscheint aber in Anbetracht der Fülle einer durchaus ernst zu nehmenden, ebenso theoretisch fundierten wie mit Erfahrungen aus der Praxis angereicherten Fachdiskussion in den USA unangemessen, den Gesamtkomplex „Public Journalism" einzig auf diesen einen Aspekt und die damit möglicherweise verbundenen Fehlentwicklungen zu reduzieren. Es dürfte daher vielmehr von Relevanz sein, auch die zukünftige Entwicklung mit wissenschaftlichen Methoden zu begleiten und kritisch zu untersuchen, wohin der Weg führt. Dafür wären sicherlich auch inhaltsanalytische Vollerhebungen eines gesamten Jahrgangs einer Zeitung - eventuell sogar im Vergleich eines nationalen und eines lokalen Blattes - aussagekräftig, um festzustellen, auf welche Weise sich „Public Journalism" dann auch in der alltäglichen Berichterstattung manifestiert, um in Kombination mit Leserbefragungen zu verlässli- 
chen Aussagen, unter anderem über den „Public Relations“-Aspekt, gelangen $z u$ können.

Bislang liegt nach Kenntnis der Autorin aufgrund der noch jungen Entwicklung nur eine einzige Studie dieser Art vor, ${ }^{14}$ die im Rahmen einer Dissertation an der Florida State University entstand. Darin wird die überregionale, in Florida erscheinende Zeitung "Tallahassee Democrat" in ihrer aktuellen Erscheinungsform sowohl mit ihrer eigenen Berichterstattung in den Jahren vor dem Aufkommen von "Public Journalism“, als auch mit einer sogenannten traditionellen Zeitung, der "Gainesville Sun“, verglichen. Brian L. Massey kommt darin zu folgenden, für die Prognose zukünftiger Entwicklungen interessanten Schlussfolgerungen: In der routinemäßigen Ausübung von „Public Journalism" kommen mehr Durchschnittsbürger als bislang zu Wort, allerdings werde die damit einher gehende, nur langsam fortschreitende Reduzierung der sogenannten „elite sources" von den Lesern offenbar noch nicht in ausreichendem Maße wahrgenommen. $\mathrm{Zu}$ durchgreifenden Veränderungen in der alltäg. lichen Berichterstattung habe "Public Journalism" in Tallahassee also zumindest in der kurzen Zeit seit seiner Etablierung im Alltags-Journalismus noch nicht geführt. „The absence of substancial, broad change in how the civic-journalism ,Democrat' deployed nonelite sources in its everyday, non-project local news may speak to the potency of traditional journalism" 15 , folgert der Untersuchungsleiter.

\section{Der Journalismus beschreitet neue Wege}

Recipes for action that could safely "connect" a newspaper with its readers sound like recipes for making omelets without breaking eggs. Such action will be either extremely messy and embarrassing ...

Max Frankel, The New York Times

14 Vgl. Massey, Brian L.: Civic Journalism and Nonelite Sourcing: Making Routine Newswork of Community Connectedness, in: Journalism \& Mass Communication Quarterly. 75. Jahrgang/Nr. 2. Sommer 1998, S. 394-407.

15 Massey, Brian L.: Civic Journalism and Nonelite Sourcing: Making Routine Newswork of Community Connectednes, in: Journalism \& Mass Communication Quarterly. 75. Jahrgang/Nr. 2. Sommer 1998, S. 102. 


\subsection{Arbeitshypothesen}

„Doing ,Public Journalism “", das heißt grob vereinfacht also im Wesentlichen:

- sich von der Tradition des ausschließlich „objektiven“, unbeteiligten Berichterstatters zu verabschieden

- Missstände nicht nur aufzudecken, sondern auch zu versuchen, zusammen mit anderen Beteiligten eine Diskussion über mögliche Lösungsansätze in Gang zu bringen

- die Leser durch Angebote wie Gemeindeversammlungen oder Telefonaktionen direkt zum Handeln aufzufordern

- den bequemen „Eliten-Journalismus" aufzugeben, bei dem man vom Schreibtisch aus zu bestimmten Fachfragen die immer wieder gleichen Experten und Politiker befragt, denn dadurch sei, so die „Public Journalism"-Pioniere, eine Sphäre der Öffentlichkeit entstanden, die nur noch auf der Ebene zwischen Journalist und Experte, vorbei am Leser, stattfinde.

Aufgrund der nach ausführlicher Literaturanalyse und Auswertung der Intensiv-Interviews gewonnenen Erkenntnisse konnten folgende deduktiv abgeleitete - Hypothesen formuliert werden:

$\mathbf{H}_{1}$ : Bei „Public Journalism“ kommen mehr Durchschnittsbürger und weniger offizielle Informationsträger zu Wort.

$\mathrm{H}_{2}$ : Durch „Public Journalism" wird das Themenspektrum der Berichterstattung erweitert.

$\mathrm{H}_{3}$ : „Public Journalism“ zeichnet sich durch deutlich mehr Meinungsbeiträge als der traditionelle Journalismus aus.

$\mathrm{H}_{4}$ : „Public Journalism" konzentriert sich bei der Wahlberichterstattung inhaltlich weniger auf die Verlautbarungen von Politikern und offiziellen Informationsträgern, sondern überwiegend auf Themen, die den Lesern wichtig erscheinen und deshalb von den Journalisten angesprochen werden.

$\mathrm{H}_{4}$ a) Dadurch werden auch Inhalte thematisiert, die nicht oder nur vereinzelt in die Zuständigkeit des zu besetzenden Amtes fallen und von daher keine Relevanz im Wahlkampf besitzen.

$\mathrm{H}_{4}$ b) Durch die überwiegende Fokussierung auf „issues" geht das "Flair" eines Wahlkampfes und somit ein wesentlicher Teil der Information über die Kandidaten verloren.

$\mathbf{H}_{5}$ : „Public Journalism“ prangert Missstände an und sucht nach konkreten Lösungsansätzen.

$\mathbf{H}_{5}$ a) Meistens werden die Problemlösungen nicht erst in der Diskussion mit den Lesern entwickelt, sondern bereits von den Journalisten vorgegeben. 
$\mathrm{H}_{5}$ b) Bei sozial-orientierten „Public Journalism“-Projekten übernehmen die Journalisten oft die Rolle von Sozialarbeitern und machen sich daher angreifbar, weil sie die Öffentlichkeit nach subjektiven Kriterien polarisieren.

$\mathrm{H}_{6}$ : Der Service-Charakter steht bei „Public Journalism“ eindeutig im Vordergrund. Das reicht von systematischen Terminankündigungen bis zur detaillierten Erläuterung komplexer Sachverhalte.

$\mathrm{H}_{7}$ : „Public Journalism“ wirkt nicht im Verborgenen, sondern informiert die Leser über seine Ideen und Ziele.

\subsection{Zusammenfassung und Interpretation der Ergebnisse}

Die sowohl quantitative als auch qualitative Überprüfung der Hypothesen anhand von insgesamt rund 500 Artikeln ergab folgende Ergebnisse: Im Rahmen der vergleichenden Wahlberichterstattungs-Analyse ließ sich zunächst eine deutliche Verschiebung im Bereich der Haupthandlungsträger feststellen. Bestimmten 1988 noch überwiegend Politiker und offizielle Informationsträger die öffentliche Agenda, so kamen 1996 wesentlich mehr, nämlich inzwischen sogar ebenso viele Durchschnittsbürger wie Politiker zu Wort, und der Anteil der offiziellen Informationsträger nahm stark ab. In Verbindung mit dem, was die „Public Journalism"-Pioniere selbst über ihre Motivation, die der Bewegung zugrunde liegt, erläutern, scheint somit ein Etappenziel auf jeden Fall erreicht: Die Berichterstattung, wie zumindest für die Wahl-Kampagnen des "Charlotte Observer" der Jahre 1988, 1992 und 1996 festgestellt werden konnte, orientiert sich ganz offensichtlich wieder mehr an den Bürgern, ihren Alltagsthemen und ihren akuten Bedürfnissen. Und da die Politiker nach wie vor in einem ebenso breiten Kontext wie die Durchschnittsbürger Eingang in die Berichterstattung der Zeitung finden, scheint sich die Befürchtung einiger Kritiker, dass mit "Public Journalism" gleichzeitig eine thematische Verlagerung vom Relevanten zum Populären stattgefunden habe, zumindest in dem prognostizierten Ausmaß nicht begründen zu lassen. Die Auswertung des erwähnten Wahlkampf-Umfeldes liefert jedoch Hinweise darauf, dass bei einer verstärkten direkten Konfrontation der Leser mit politischen Kandidaten und somit auch in der Berichterstattung darüber durchaus die Gefahr besteht, dass nebensächliche - beispielsweise persönliche - Themen einen ihnen völlig unangemessenen Raum einnehmen. Wird also, und darin liegt nun die nach Einschätzung der Autorin erste deutliche Gefahrenquelle in der „Public Journalism“-Berichterstattung, die Themenauswahl nicht mehr durch die nach professionellen Kriterien entscheidende "Gatekeeper-Station" der Journalisten gefiltert, so könnte das - wie auch in 
einigen Interviews, insbesondere in den Aussagen von Politik-Redakteur Dan Chapman, deutlich wurde ${ }^{16}-\mathrm{zu}$ einer in dieser Ausprägung wohl kaum erwünschten Trivialisierung führen. Dass es im Falle der Wahlberichterstattung offensichtlich aber nicht so weit kam, dafür spricht die Auswertung der Agenda-Setter-Daten. Dabei zeigt sich, dass auch unter den Einflüssen des „Public Journalism" in den meisten Fällen die Journalisten als Agenda-Setter auftraten, sich jetzt aber im Gegensatz zu früher dadurch, dass sie vielfach Durchschnittsbürger zu Haupthandlungsträgern ihrer Artikel machen, deren Themen zu eigen machen.

Anhand der aufgezeigten Themenauswahl im Jahresvergleich lässt sich von 1988 bis 1996 - das Jahr 1992 als Zwischenstation einmal ausgeklammert - zwar nur eine geringfügige Erweiterung des Themenspektrums belegen, interessanter erscheint jedoch der deutlich angestiegene prozentuale Anteil der so genannten sach- und problemorientierten Themen, während die rein auf den Wahlkampf also solchen bezogene Berichterstattung erheblich zurückging. Das spricht bei einem zahlenmäßigen Anstieg der im Wahlkampf erschienenen Artikel dafür, dass unter den Vorgaben des „Public Journalism“-Gedankens eine Form der journalistischen Präsentation gewählt wurde, die weg vom Spotlight-artigen Anriss möglichst vieler Themen bei noch leicht zunehmender Anzahl den einzelnen Problemfeldern durch vielfältigere Berichterstattung mehr Platz einräumt und somit sowohl mehr Hintergrundinformation als auch ein breiteres Meinungsspektrum zur Verfügung stellt.

Im weitesten Sinne zur Erweiterung der Hintergrund-Berichterstattung zählt auch die enorme Zunahme von Artikeln mit ankündigender Funktion, sei es als Hinweis auf einen Folgeartikel, sei es auf eine Veranstaltung, die meistens in einem inhaltlichen Kontext mit der Berichterstattung steht. Der Service-Charakter im „Public Journalism“, zusätzlich journalistisch artikuliert in einer verstärkt mit Bildern und insbesondere erklärenden Grafiken angereicherten Themen-Präsentation, wird sowohl durch die Analyse der Wahlberichterstattung als auch die beiden SozialProjekte untermauert. Im Falle von „The People Project" sowie "Taking back our neighborhoods" erfüllen insbesondere die überwiegend sonntags erscheinenden themenbezogenen Packages in Form vielfältiger journalistischer Darstellungsformen diese Funktion.

Während sich bei der Wahlberichterstattung der Schwerpunkt der Haupthandlungsträger im Rahmen von „Public Journalism" von Politikern und offiziellen Informationsträgern in Richtung der Durchschnittsbürger verlagerte, treten beiden Untersuchungseinheiten neben den dar-

16 Vgl. Dan Chapman im Gespräch mit der Autorin, a.a.0., siehe Anhang, S. 216. 
unter fallenden Arbeitern, Angestellten, Hausfrauen, Schülern, Lehrern und anderen mehr zusätzlich auch soziale Außenseiter wie die der sogenannten Normabweichler - Drogenabhängige/-dealer, Arbeitslose, Analphabeten, Kranke und Behinderte beispielsweise -, oder Bürgerinitiativen auf. $\mathrm{Da}$, wie insbesondere im Interview mit Jim Walser deutlich wurde, diese Randgruppe wohl kaum als neue Abonnenten-Zielgruppe in Frage kommt, lässt sich die Schlussfolgerung ableiten, dass durch die Ausweitung der allgemeinen Berichterstattung auch auf solche, problembeladene und mit sozialem Sprengstoff versehene Themen die allgemeine Glaubwürdigkeit der Zeitung bei der bestehenden Leser-Klientel gestärkt werden soll.

Bei dieser Art der Berichterstattung übernehmen die Journalisten zumindest im Fall der vorliegenden Untersuchungen - im Gegensatz zu häufig angefïhrter Kritik jedoch nicht die Rolle von Sozialarbeitern, sondern bewegen sich nach wie vor, wenn auch in stärkerem Maß als bisher mit anwaltschaftlicher, ratgebender, kommentierender und interpretierender Funktion, im Bereich des klassischen Spektrums journalistischen Rollenverständnisses.

Allerdings konnte, und hier verbirgt sich nach der Auffassung der Autorin die zweite nicht zu unterschätzende Gefahr bei der praktischen Anwendung der „Public Journalism“-Idee, bei der Serie „Taking back our neighborhoods" anhand der vorliegenden Artikel nachgezeichnet werden, dass die Journalisten in der überwiegenden Zahl der Fälle mögliche Lösungsansätze nicht nur thematisierten und den Lesern zur Diskussion stellten, sondern gleich ein fertiges Konzept - und wenn es auch nur aus einer einzelnen Handlungsaufforderung bestand - darlegten, zu dem es keine sinnvollen Alternativen zu geben schien. Geht man als Journalist soweit, dann führt man allerdings den eigentlichen Grundgedanken des "Public Journalism" ad absurdum, indem man die Leser zwar als Handlungsträger einbezieht, eine öffentliche Diskussion, wie sie sowohl im Sinne von Habermas, als auch von John Dewey, Jay Rosen oder den Kommunitaristen ausdrücklich gefordert wird und in einer re-politisierten Gesellschaft auch das Ziel des „Public Journalism" sein sollte, durch die Vorgabe einer fertigen Lösung jedoch bereits im Keim erstickt. Abschließend soll nun, wie bereits in der Einleitung skizziert, eine gedankliche Brücke geschlagen und der Frage nachgegangen werden, in wieweit sich Parallelen zwischen der Entwicklung der „Public Journalism"-Bewegung in den USA und neuen redaktionellen Konzepten in Deutschland aufzeigen lassen. 


\section{Der „Public Journalism"-Gedanke im deutschen Journalismus}

Von dem ganzen Journalismus ist in diesem Augenblick nur derjenige wirklich lebendig und wird nur noch der von dem Interesse der Lesenden wirklich getragen, der sich näher oder ferner, referierend oder raisonnirend, bekämpfend oder zustimmend, auf die politischen Zustände unserer Gegenwart einläßt. Der übrige Journalismus ist todt oder führt nur ein Scheinleben, dem es an Wahrheit wie an Kraft gebricht.

Robert Prutz 1845

Das Berufsverständnis des Journalisten als aktiver Faktor in der Gesellschaft hat in Deutschland im Gegensatz zur „Public Journalism"-Bewegung in Amerika eine jahrhundertelange, über Zensurvorschriften und andere Repressalien hinweg sich zwar verändernde, aber doch im Grundgedanken bewahrte Tradition. Im Angelsächsischen bildete sich dagegen ein - im deutschen Journalismus immer nur sporadisch auflebendes Konzept der Unparteilichkeit heraus, das mit seinen typischen Charakteristika der Funktion des Journalismus in einer komplexen Gesellschaft entspricht, die bei ihrer sozialen „Kommunikation auf Distanz" auf die Vermittlung durch hochrationalisierte, spezialisierte Vermittlungs-Institutionen, die Massenmedien, angewiesen ist. Gibt der Journalist seine spezifische, unparteiliche Haltung auf, verlässt er zugleich die professionelle Vermittlungsrolle und übernimmt die Rolle einer der "Parteien“ innerhalb der sozialen Kommunikation anstatt alle zu vermitteln. ${ }^{17}$

In Anbetracht jüngster Umfrageergebnisse zur Leser-Blatt-Bindung wie auch unter Berücksichtigung vorliegender Kommunikator-Studien der vergangenen Jahre, die die Annahme einer sozialen Distanz zwischen den Journalisten und ihrem Publikum als gesichert ansehen, ${ }^{18}$ wird nach einer Phase Anfang der 90-er Jahre, in der sich auch der überwiegende Teil der deutschen Journalisten vor allem als neutrale Vermittler verstanden (74,1 Prozent), ${ }^{19}$ inzwischen unter dem ökonomischen Druck stagnierender Auflagenzahlen nach neuen redaktionellen Konzepten

17 Vgl. Schönhagen, Philomen: a.a.0., S. 292.

18 Vgl. Weischenberg, Siegfried: Der enttarnte Elefant. Journalismus in der Bundesrepublik - und die Forschung, die sich ihr widmet, in: Media Perspektiven 4/89, S. 232.

19 Vgl. Weischenberg, Siegfried/Löffelholz, Martin/Scholl, Armin: Merkmale und Einstellungen von Journalisten. Journalismus in Deutschland II, in: Media Perspektiven 4/94, S. 160. 
Ausschau gehalten, die gleich mehrere Zwecke erfüllen sollen: Zum einen geht es darum, die auch in Deutschland entstandene Kluft zwischen Journalisten und Lesern wieder zu schließen, zum anderen die Glaubwürdigkeit des Mediums Zeitung langfristig zu erhöhen und letztlich durch eine weniger an den sogenannten Eliten oder "opinion leadern" ausgerichtete Verlautbarungs-Berichterstattung, sondern durch serviceorientierte, für die Leser im Alltag relevante Themen mehr Menschen an die Zeitung zu binden und dem Blatt dadurch auch in Zukunft seinen Platz als festem Bezugspunkt in der Gemeinde zu sichern. Was in dieser Hinsicht während der 70-er Jahre vom politischen Zeitgeist inhaltlich gestaltet vorangetrieben wurde, wird heute aus eher wirtschaflichen Erwägungen zur Neuauflage mit leicht veränderten Vorzeichen herangezogen.

In Anbetracht all dieser Indizien kann im Rahmen dieser Zusammenfassung jetzt auch auf die letzte der zwölf eingangs gestellten Forschungsfragen eine auf aussagefähigem Material fußende Antwort gegeben werden: So sehr "Public Journalism“ sich aus Sicht der amerikanischen Wissenschaftler und Redaktionspraktiker auf den ersten Blick als ein rein amerikanisches Phänomen ausnimmt, so deutlich kristallisieren sich bei vertiefter Betrachtung doch eindeutige inhaltliche Parallelen $z u$ Entwicklungen und ähnlichen Problemlösungs-Ansätzen heraus, wie sie in der deutschen Journalismusgeschichte bereits mehrfach in jeweils unterschiedlicher Ausprägung und Intensität unternommen worden sind. Es handelt sich daher - in Anlehnung an $F_{12}$ - bei der zuvor ausführlich geschilderten, der gesamten Bewegung zugrunde liegenden Idee wie auch ihrer Umsetzung wohl kaum um eine rein amerikanische Entwicklung. Vielmehr greift ja bereits Jay Rosen in seiner theoretischen Begründung auf europäisch-philosophische Ideengeschichte zurück, setzt sie mit typisch amerikanischen Gesellschaftsphänomenen in Bezug, und öffnet das fertige "Produkt" in Form bewährter Projekte auf internationalen Fachkongressen dem Zugriff, der Nachahmung und Weiterentwicklung durch ein Publikum weltweit agierender Journalisten.

\section{Schlussbetrachtung}

In committing an act of public journalism, you know you have succeeded when you have left behind something people continue to use, some added ability the community now posses. The power of the press thus empowers others besides the press. 
Mit "Public Journalism" erregte Anfang der 90-er Jahre in den USA ein neues journalistisches Konzept die Aufmerksamkeit der Journalisten, da es für die auf Objektivitäts-Anpruch und investigative Recherchemethoden geschulten Redakteure zunächst ein ihren Vorstellungen völlig entgegengesetztes Berufsverständnis zu propagieren schien: Anwalt statt Kritiker, Propagandist statt neutraler Beobachter sollten sie nun plötzlich sein, und sich - zuvor allenfalls dem wirtschaftlichen Wohlergehen des jeweiligen Verlagskonzerns verpflichtet - in den Dienst der Demokratie stellen, um ein sowieso nur mäßig interessiertes Publikum für verstärktes politisches Engagement zu gewinnen. Der Widerstand in den meisten Redaktionen gegen ein solches Konzept war groß, und diejenigen, die in Wichita, Madison, Charlotte und anderswo erste "Gehversuche" auf dem glatten Parkett des neuen Rollenverständnisses wagten, ernteten Kritik von allen Seiten.

Von den Kollegen, weil die Wiederherstellung einer - anerkanntermaßen verlorengegangenen - Verbindung zu den Lesern, mit verstärkter Recherche und somit häufigeren Außenterminen verbunden, ein größeres Engagement seitens der Journalisten voraussetzte, und von den bislang als Hauptinformationsträger fungierenden gesellschaftlichen „Eliten" wie offiziellen Informationsträgern von Behörden, Verbänden und Betrieben sowie einer nicht geringen Anzahl von Politikern, die nun befürchteten, dass ihre Interessen bei einer verstärkt auf die Interessen der "Durchschnittsbürger" ausgerichteten Berichterstattung in nicht mehr ausreichendem Maße Niederschlag in den Artikeln ihrer Lokalzeitung finden würden. Die einzigen, die - verlässt man sich auf die Aussagen der an „Public Journalism“-Projekten beteiligten Journalisten sowie den in diesem Zusammenhang abgedruckten Leserbriefen - diese Entwicklung spontan für gut befanden, waren die Menschen in der Stadt und darunter insbesondere die sozialen Randgruppen, deren Stimme jetzt auch $\mathrm{ab}$ und $\mathrm{zu}$ bis in die Redaktionsräume vordrang, und mit deren Bedürfnissen, die nun verstärkt auf die öffentliche Agenda kamen, sich die Gemeinschaft daraufhin auseinanderzusetzen hatte. Denn was zunächst in zaghaften Schritten im Rahmen der Wahlkampf-Berichterstattung als zeitlich begrenztes Unternehmen versuchsweise gestartet worden war, entwickelte trotz anhaltender kritischer Stimmen eine Eigendynamik, die in teilweise über mehrere Jahre andauernden, sozialorientierten Berichterstattungs-Serien ihren Ausdruck fand.

Einige Verleger witterten bald die Chance, mit „Public Journalism“ quasi gratis - denn personelle Mehrkosten oder gar redaktionelle Umstrukturierungen fanden nur in den seltensten Fällen statt - eine neue Public-Relations-Strategie an die Hand zu bekommen, mit der die seit Jahren anhaltenden Auflagenschwunde abgefangen werden könnten. 
Doch zur allgemeinen Enttäuschung der Verleger ist der erwünschte Effekt zumindest in der bislang zehnjährigen Geschichte der „Public Journalism"-Bewegung nicht eingetreten. Statt dessen schossen manche Projekte weit übers Ziel hinaus, und viele Journalisten spürten nicht selten die Gefahr, in vermeintlich guter Absicht zum Sprachrohr gezielt verfolgter Einzel-Interessen gemacht zu werden.

Inzwischen sind die anfangs notwendigen Ecken und Kanten, um überhaupt Aufsehen erregen und etwas bewegen zu können, weitgehend abgeschliffen, und „Public Journalism" hat sich vom provokanten projektbezogenen Aktivismus zu einer weitestgehend anerkannten Berufsphilosophie entwickelt. Kostenintensive Unternehmungen wie das Veranstalten von TV-Diskussionsrunden oder das Sponsoring von Freizeitstätten für Jugendliche wurden in den meisten Fällen aufgegeben zugunsten einer aufgeschlosseneren, stärker an den von den Lesern artikulierten Bedürnissen orientierten Berichterstattung im Alltag.

Sicherlich besteht nach wie vor die Gefahr, im Zeichen des „Public Journalism" bestimmten Gruppierungen „nach dem Mund zu reden“. Zudem zeigte sich anhand der inhaltsanalytischen Untersuchung, dass die Tendenz der Journalisten besteht, Lösungsansätze, über die sie sich jetzt verstärkt Gedanken machen, aber gar nicht erst mit den Lesern zu diskutieren, sondern in der Berichterstattung bereits fertige Konzepte zu präsentieren. Damit allerdings hätte man - wie bereits an anderer Stelle erwähnt - den Kerngedanken der „Public Journalism“-Bewegung, nämlich das Anregen einer offentlichen Diskussion in einem zumindest graduell re-politisierten Klima eines überschaubaren, stadtteilbezogenen Umfeldes aufgegeben, noch bevor er sich überhaupt entfalten konnte.

Es wäre sicherlich verfehlt, in „Public Journalism“ eine grundsätzliche Umwälzung des gesamten amerikanischen Journalismus erkennen zu wollen. Aber zumindest hat er dafür gesorgt, dass mittlerweile auch renommierte Blätter wie die „Washington Post" oder die „New York Times" ihre Berichterstattung selbstkritisch unter die Lupe nehmen und nach Verbesserungsmöglichkeiten suchen, was in der vorliegenden $\mathrm{Di}$ plomarbeit zwar nicht genauer untersucht werden konnte, sicherlich aber interessante Ansätze für weitere Forschungsarbeiten in dieser Richtung liefern könnte.

Eines aber hat "Public Journalism", in Amerika und anderswo, ob mit oder ohne diesen Namen, zweifellos erreicht: Die Ideen von Jay Rosen, Davis Merritt und ihren vielen, unermüdlichen Mitstreitern führten zu einem grundsätzlichen Nachdenken und Infragestellen einiger gängiger Methoden im amerikanischen Redaktionsalltag und einer zumindest in Ansätzen stattfindenden Rückbesinnung auf so manche berufsethische Maxime wie beispielsweise den grundsätzlichen Gegen-Check der einge- 
holten Informationen oder die Reporter-Tugend der Vor-Ort-Recherche. Selbst wenn manche Journalisten, die sich monatelang den Kopf darüber zerbrachen, was „Public Journalism“ ihnen alles abverlange, am Ende zu der Auffassung gelangen, dass das unter diesem Label Propagierte nichts anderes sei als "schlichtweg guter Journalismus", dann scheint im Zeitalter der Kommerzialisierung und Trivialisierung des allgemeinen Medienangebotes schon viel gewonnen.

Das Problem der traditionellen Berufsnormen, so stellte Wolfgang Donsbach vor nicht allzu langer Zeit fest, bestehe darin, "dass sie für den Berufsalltag kaum Handlungsanleitungen bieten". ${ }^{20}$ Er schlägt, um dieses Problem zumindest ansatzweise zu lösen, „Pluralismus“ als Zentralwert vor. „Als konkrete Norm ließe sich daraus [...] ableiten, dass das Mediensystem jedem einzelnen Bürger eine möglichst vielfältige Wirklichkeitsdarstellung bieten sollte, die weitgehend unbeeinflusst von Interessengruppen und subjektiven Vorlieben der Medien und ihrer Macher ist." 21 Als additiv ergänzendes, keineswegs losgelöst von anderen Berufsnormen anzuwendendes Modell verantwortungsvoller Berichterstattung will die Autorin aufgrund ihrer vorliegenden empirischen Bestandsaufnahme das Konzept „Public Journalism“ verstanden wissen; als Handlungsmaxime, der in einer von Politikverdrossenheit und mangelnder Bürgerbeteiligung geprägten Atmosphäre für die Erhaltung einer freiheitlichen Staatsform besondere Bedeutung zukommt, da der Demokratie genau dann Gefahr droht, wenn die soziale Kommunikation "unvermittelt bleibt und das demokratische Gesellschaftsplenum keine sozialuniversellen Medien vorfindet, um im allseitigen Austausch der Tatsachen und Überzeugungen jene am Gemeinwohl orientierten politischen Kompromisse zu finden und zu schließen, welche die Demokratie tragen und programmieren." 22

20 Donsbach, Wolfgang: Der Pluralismus als journalistische Norm. Neuansatz einer grundlegenden, bisher oft missverstandenen Medienethik, in: TM. Transparenz und Medien. 6/1996, S. 9.

21 Ebenda.

22 Starkulla, Heinz: Marktplätze sozialer Kommunikation. Bausteine einer Medientheorie, München 1993, S. 71. 